\title{
Hopping-Pong: Changing Trajectory of Moving Object Using Computational Ultrasound Force
}

\author{
Tao Morisaki ${ }^{1}$, Ryoma Mori $^{2}$, Ryosuke Mori ${ }^{3}$, Yasutoshi Makino ${ }^{4}$, Yuta Itoh ${ }^{5}$, \\ Yuji Yamakawa ${ }^{6}$, Hiroyuki Shinoda ${ }^{7}$ \\ The University of Tokyo ${ }^{1,2,3,4,6,7}$, Tokyo Institute of Technology ${ }^{5}$, JST PREST ${ }^{4,5,6}$ \\ Kasiwa-shi ${ }^{1,2,3,4,7}$, Yokohama-shi ${ }^{5}$, Meguro-ku' ${ }^{6}$, Japan

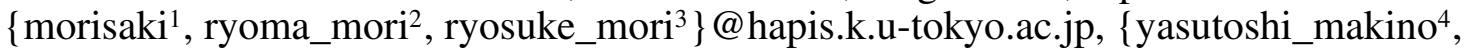

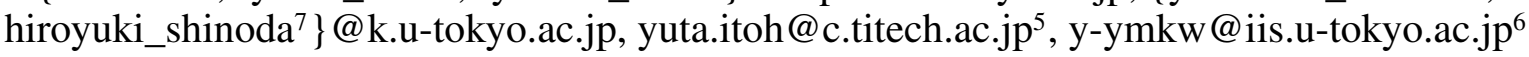
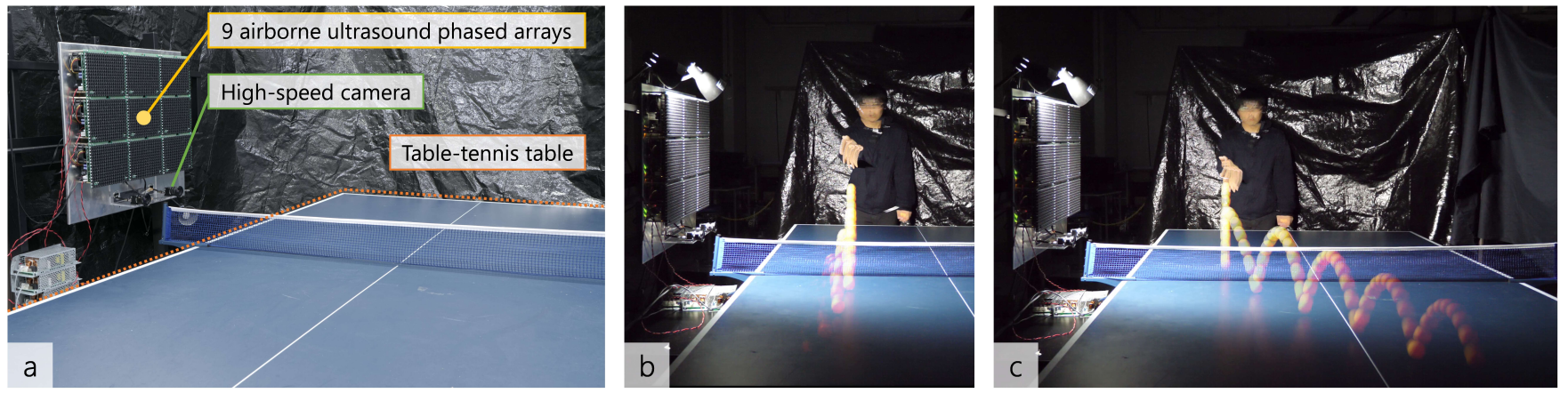

Figure 1. Our dynamic force presentation system: Hopping-Pong. (a) A side view of our system configuration. (b) A snapshot of throwing ping-pong ball. (c) A snapshot of a scene where our system dynamically modifies the trajectory of a ping-pong ball. Each photo is a merged image created from each throwing trial to visualize ball trajectories.

\section{ABSTRACT}

Physically moving real objects via a computational force connects computers and the real world and has been applied to tangible interfaces and mid-air display. Many researchers have controlled only a stationary real object by computational force. On the other hand, controlling a moving object can expand the real space that is controllable by the computer. In this paper, we explore the potential of computational force from the viewpoint of changing the trajectory of a moving object. Changing the trajectory is the primitive model to control a moving object, and it is the technological challenge requiring high-speed measurement and non-contact force with high-spatial resolution. As a proof-of-concept, we introduce Hopping-Pong changing the trajectory of a flying Ping-Pong B all (PPB) u sing ultrasound force. The result shows that Hopping-Pong changes the trajectory of a PPB $344 \mathrm{~mm}$. We conclude that a computational force is capable of controlling a moving object in the real world. This research contributes to expanding the com-

Permission to make digital or hard copies of part or all of this work for personal or classroom use is granted without fee provided that copies are not made or distributed for profit or commercial advantage and that copies bear this notice and the full citation on the first page. Copyrights for third-party components of this work must be honored. For all other uses, contact the owner/author(s).

ISS '19, November 10-13, 2019, Daejeon, Republic of Korea.

Copyright is held by the author/owner(s)

ACM ISBN 978-1-4503-6891-9/19/11.

http://dx.doi.org/10.1145/3343055.3359701 putationally controlled space with applications for augmented sports, HCI and factory automation.

\section{CCS Concepts}

-Human-centered computing $\rightarrow$ Human computer interaction (HCI); Interaction devices;

\section{Author Keywords \\ Controlling Moving Object;Computational \\ Force;Ultrasound;Augmented Ping-Pong;}

\section{INTRODUCTION}

Connecting computers and the real world is one of the fundamental motives driving our research community. Physically moving real objects via a non-contact computational force presentation is at a unique end of this research spectrum. Different works employ different mediums for force generation including magnetic [16], ultrasound [31], and air-jet [3]. Researchers have also proposed applications such as a mid-air display using particles like 3D pixels [25] and a tangible interface [19]. While these works allow us to manipulate physical objects in mid-air using computers, they focus only on manipulating stationary objects. This limits the application space mainly to displays as we introduced above.

Thus, in this study, we explore the potential of computational force generation by applying to change the future trajectory of a real moving object in the environment where computational force devices are embedded. Changing trajectory is the 
primitive model to control a moving object. We believe the concept expands the application space in related fields such as augment sports, HCI, and factory automation technology.

Changing the trajectory of moving object is the technological challenge because of the requirements: 1) high-speed threedimensional measurement, 2) non-contact force presentation with high spatial resolution and high frame rate, 3) combining the measurement with the force presentation while maintaining high frame rates, 4) presenting force enough to change the trajectory totally. Additionally, the computational force system must track a moving object and keep applying force only to it with a computational force whose presentation area is smaller than a moving object. For example, a force presentation to the large area e.g. a big air blower stimulates not only a moving object but also its surroundings.

As a proof-of-concept system, we introduce a computational ultrasound force system called Hopping-Pong. This system measures the trajectory of a Ping-Pong Ball (PPB) flying in mid-air by a high-speed two cameras and changes it through a non-contact ultrasound force. Ultrasound can creates a small stimulation area that is approximately $1 \mathrm{~cm}^{2} \times 3 \mathrm{~cm}$ and smaller than a PPB $[10,5]$. In addition, the stimulation position can be controlled with a frame rate of $1 \mathrm{kHz}$ [11]. This spatiotemporal resolution is higher than air-jet [3]. Therefore, we conjectured that ultrasound is suitable for high-speed tracking a moving object without stimulating surroundings. Combined with a high-speed measurement using a stereo camera, the small stimulation area dynamically and three-dimensionally tracks a PPB. Our evaluation shows that Hopping-Pong successfully changes the trajectory of a moving PPB. This demonstrates that our concept is capable in environment where ultrasound force devices are embedded.

Hopping-Pong itself also stands as a potential application of our concept to illustrate the non-contact force that dynamically tracks moving object with high three-dimensional spatial resolution in computer game-like entertaining sports. Ultimately, all players under the system could hit a super-shot. In addition to this, by controlling the timing to change the trajectory of a PPB, the system enables us to design a game that can balance the physical ability or experience differences of each user.

Since the non-contact force tracking system, including Hopping-Pong, controls the trajectory of a moving object it has potential applications in industry such as removing impurities in factories. Such a system can selectively stimulate and remove specific objects.

\section{Contributions}

Our main contributions include the followings

- We describe the concept of controlling the trajectory of a moving object using non-contact force

- We demonstrate a proof-of-concept system: Hopping-Pong, combining an ultrasound force generator and a high-speed measuring system

- This research expands the real space that is controllable by computers and allows us to propose many applications

\section{RELATED WORKS}

\section{Controlling Real Object}

There are many methods to control real objects such as using magnetic [16], air-jet [3], and ultrasound [31, 6, 1, 12]. Many researchers have focused on levitation and manipulation using ultrasound. The following points elaborate on this.

Ochiai et al. three-dimensionally manipulated particles trapped by the standing wave with an ultrasonic phased array facing each other [26]. The method of using a standing wave is traditional and robust [31], but this approach can only trap an object that is smaller than the half wavelength of the ultrasound. Many researchers proposed levitating or manipulating objects which is larger than the half wavelength of the ultrasound using ultrasound. Foresti et al. levitated and manipulated a wire that is longer than the wavelength by modulating an acoustic potential field spatiotemporally [6]. Andade et al. levitated polystyrene spheres of $50 \mathrm{~mm}$ in diameter [1] and curved a rectangle of $2.3 \mathrm{~g}$ [2] by creating a standing wave between the transducer and the object. This method used acoustic emission pressure. Frumoto et al. have three-dimensionally manipulated a lightweight balloon using ultrasound plane wave [9]. Inoue et al. proposed an acoustical boundary hologram and by using the hologram, they levitated a non-rotating object of $50 \mathrm{~mm}$ in diameter and $500 \mathrm{mg}$ in weight at a position away from the transducers [12].

\section{Applying Levitation and Manipulation to $\mathrm{HCl}$}

Computational control of real objects has realized many tangible interfaces. Berkelman et al. presented haptic feedback by using a magnetically floating sphere [4]. Lee et al. proposed Zero-N [18], this allows users to interact with computer systems by manipulating a magnetically floating sphere by hand. Marshall et al. proposed Ultra-tangibles [19] that twodimensionally manipulates sphere objects by surrounding the periphery of the spheres with ultrasound transducers. Ultratangibles can display tangible information. VI et al. proposed Tastyfloats [30] that is a non-contact food delivery system using ultrasound. Tastyfloats manipulates small food and directly delivers it to the user's mouth. Freeman et al. showed that accurate pointing to floating objects is possible by shaking the pointed object as a form of feedback [7].

Controlling the motion of real objects can also reproduce biological movement. Kono et al. proposed Lapillus bug [17], which moves particles like a bug. The particles are controlled by ultrasound. Yui et al. proposed Floatio [32] that is a floating ball that gives an animacy impression. Floatio is controlled by air-jet.

In addition, control of real objects is applied to tangible graphics display. Ochiai et al. proposed Pixie Dust [25] projecting an image onto particles that are levitated and manipulated by ultrasound. Furumoto et al. proposed Baluna [8] projecting an image on a floating balloon manipulated by ultrasound. Uno et al. proposed Luciola [29], a luminous body of $3.5 \mathrm{~mm}$ in diameter and $16.2 \mathrm{mg}$ in weight that floats using ultrasound. Luciola is powered wirelessly and users can touch Luciola with the hand. Sugiura et al. proposed Graffiti Fur [27], computationally drawing a re-drawable picture on a carpet by 


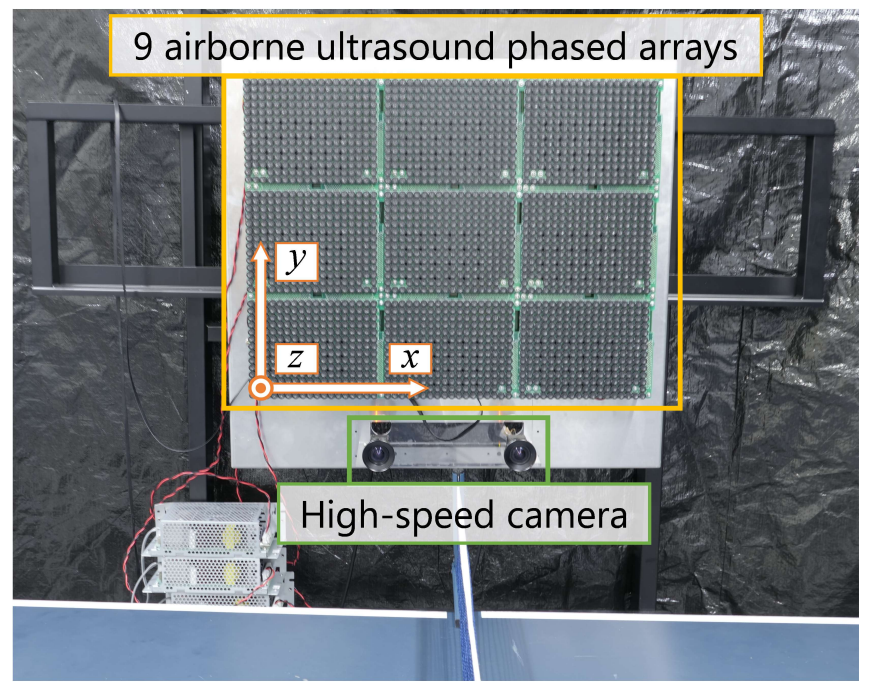

Figure 2. Front view of Hopping-Pong. In Hopping-Pong, the coordinate system is a right-handed system with an origin at the lower left of nine ultrasound phased arrays.

manipulating the furs of the carpet surface using ultrasound. Marzo et al. proposed an interactive sand canvas called Ghost Touch [20]. This copies and draws a picture originally drawn by users with ultrasound. Ghost Touch can control the diffusion of liquid, so it can draw a picture on a milk surface using the dropped color ink.

\section{Augmented Sports}

Interaction between computers and users has the potential to realize an augmented sport that can be enjoyed regardless of experience or physical ability. Ishii et al. proposed PingPongPlus [13]; this involves projecting interactive images according to a motion of a PPB on a table-tennis table. Itoh et al. proposed Laplacian Vision $[14,15]$, which allows users to see the expected trajectory of a PPB through Mixed Reality glass. Mueller et al. proposed a new ping-pong-like game that allows users to play even if they are geographically separated from each other by using a video conference system and a projector [21,22]. Nojima et al. added a computer-game-like parameter such as life-point [24]. In this system, when a ball hits a user, the user's life points decrease. Nitta et al. proposed HoverBall [23] that incorporates a drone into the ball to control the trajectory of a moving ball and suspend it in mid-air. HoverBall uses built-in actuators to control the trajectory of the moving object. On the other hand, Hopping-Pong aims at passive control only by external force.

\section{IMPLEMENTATION}

In this section, we present the implementation of our HoppingPong system. Hopping-Pong is a system that tracks the trajectory of a moving PPB in mid-air using two high-speed cameras and changes its trajectory with ultrasound.

\section{System Configuration}

The system configuration is shown in Figure 1 (a) and Figure 2. Hopping-Pong consists of nine Airborne Ultrasound Phased Array units (AUPA, shown in Figure 3) $[10,11,28]$ that apply

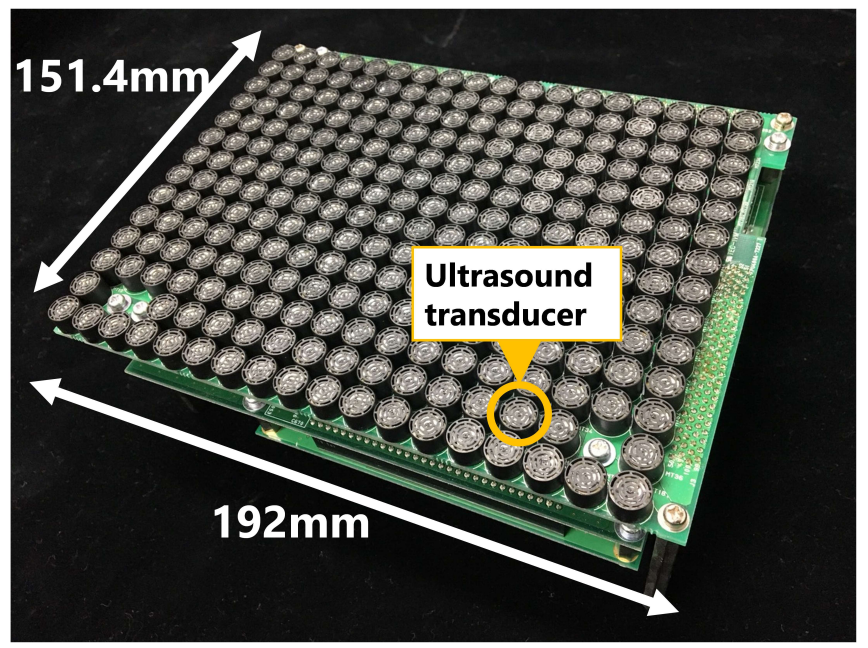

Figure 3. Single airborne ultrasound phased array unit.

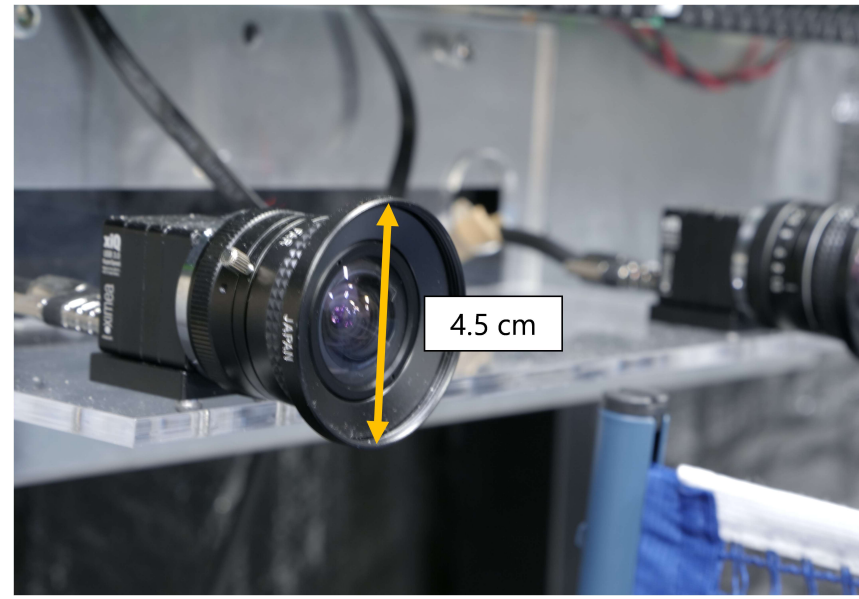

Figure 4. High-speed camera.

force to a PPB, two high-speed cameras (Ximea MQ003CGCM, shown in Figure 4) that track the PPB, and a Windows PC that controls them.

In Hopping-Pong, the coordinate system is a right-handed system with an origin at the lower left of nine AUPAs as shown in Figure 2.

\section{Measuring Three-Dimensional Position of Ping-Pong Ball Using High-Speed Camera}

Hopping-Pong measures a three-dimensional position of a PPB using two calibrated high-speed cameras. To calibrate, we used Levenberg-Marquardt algorithm. Figure 5 shows the flow of detecting a PPB. First, the system detects the pixel position of a PPB in each of the captured images using a binarization color filter. The pixel position is calculated from the center of gravity of the binarized image. The binarization range is from $(10,170,60)$ to $(40,220,180)$ that is HSV value. The parameter was turned manually. Second, the pixel positions are converted to the two-dimensional positions of the PPB in the real world by calibration parameters. Finally, the threedimensional position of the PPB is obtained by triangulation. 
Paper Session 3: Ultrasonic Techniques and Mid-air Displays
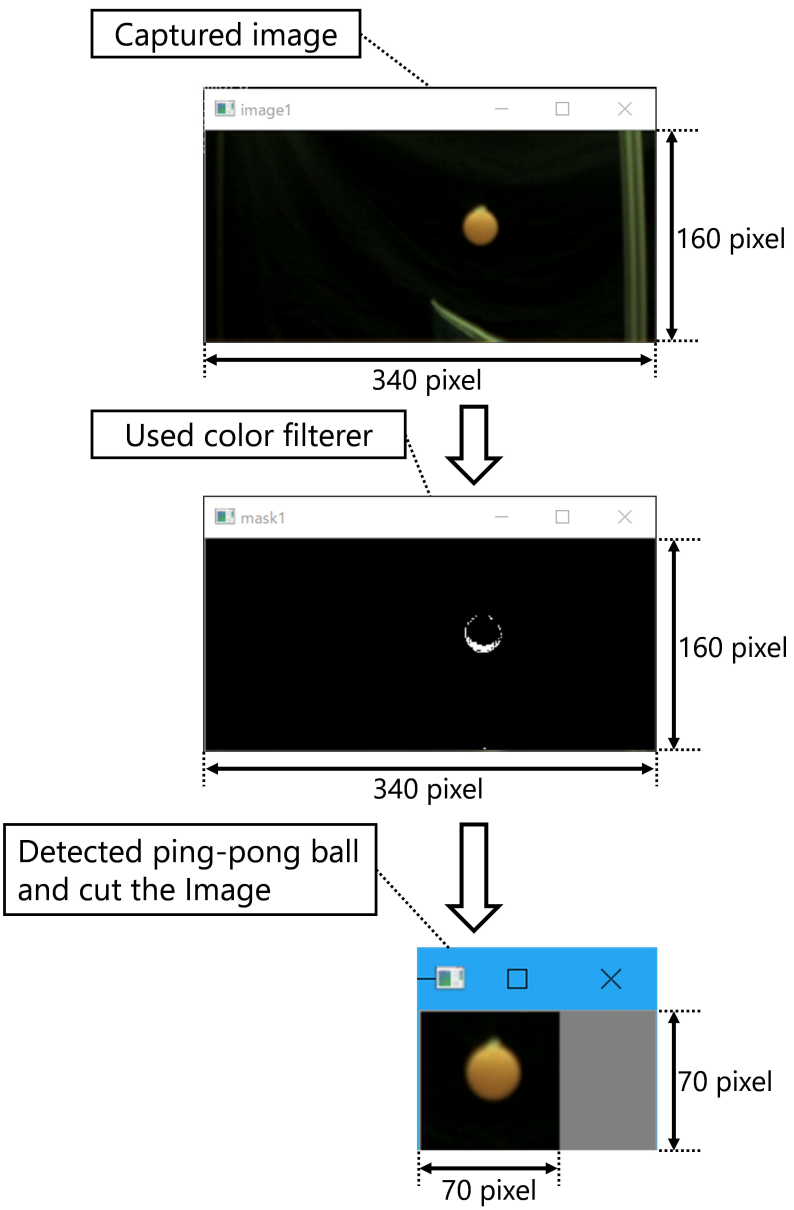

Figure 5. Detection flow of ping-pong ball.

Improving frame rate by limiting processing range

The high-speed cameras can capture up to $500 \mathrm{fps}$. HoppingPong system's frame rate is $200 \mathrm{fps}$ because of the time required for image processing and driving AUPA units. To reduce processing time as much as possible, we reduced the camera's view area from $640 \times 480$ pixel to $340 \times 160$ pixel in hardware. Moreover, after finding a PPB, the system trims the range of $70 \times 70$ pixel centered on the PPB and processes only as shown in Figure 5.

\section{Evaluation of measuring accuracy}

In this experiment, we evaluated the accuracy of measuring the three-dimensional PPB's position by two calibrated highspeed cameras. The measured PPB was manipulated and suspended in each measurement point by a three-axis stage. Figure 6 shows the experimental setup. The experimental procedure was as follows: 1) We attached a PPB to the threeaxis stage so that it could be seen from the camera. 2) The PPB moved along the $\mathrm{x}, \mathrm{y}$, and $\mathrm{z}$-axis by $35 \mathrm{~mm}$ respectively by manipulating the three-axis stage. The PPB moved totally $70 \mathrm{~mm}$ along each axis thus, the number of the measurement points is $3 \times 3 \times 3=27$. 3) The tracking system measured and recorded a three-dimensional position of the PPB at each measurement point.
ISS'19, November 10-13, 2019, Daejeon, Republic of Korea

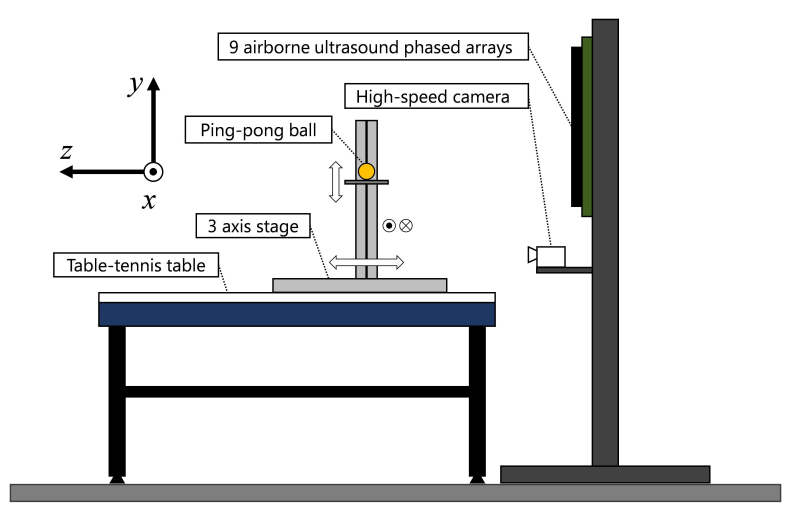

Figure 6. Experimental setup to evaluate tracking accuracy of ping-pong ball (PPB) by stereo high-speed cameras. A PPB is threedimensionally manipulated and suspended in each measurement point by a three-axis stage.

Figure 7 (A) shows the experimental results plotted on the $x-y$ plane and Figure 7 (B) shows it plotted on the $z-y$ plane. The mean value of error in the $\mathrm{x}$-axis is $0.72 \mathrm{~mm}$, in the $\mathrm{y}$-axis it is $0.76 \mathrm{~mm}$, and in the $\mathrm{z}$-axis it is $1.96 \mathrm{~mm}$. The diameter of the PPB is $40 \mathrm{~mm}$ and that of the ultrasound high-pressure point is $10 \mathrm{~mm}$ [10]. Therefore, the tracking accuracy is enough for the high-pressure point to hit a PPB.

\section{Non-Contact Force by Ultrasound Airborne Ultrasound Phased Array}

In Hopping-Pong, we used nine Airborne Ultrasound Phased Array (AUPA) units [10] to present a computational noncontact force. One unit of AUPA which we used is provided with 248 individually controllable ultrasound transducers. By controlling the phase so that sound wave focuses at a single point, AUPA creates a small high-pressure point in mid-air. The high-pressure point is approximately $1 \mathrm{~cm}^{2} \times 3 \mathrm{~cm}$ and smaller than a PPB [10]. This mechanism is illustrated in Figure 8 (A) and a distribution of effective sound pressure $p \mathrm{~Pa}$ is shown in Figure $8(\mathrm{~B})$. This distribution is normalized theoretical calculation when using square AUPA provided $50 \times 50$ transducers. The high-pressure point is created at $z=200 \mathrm{~mm}$ and $z=0 \mathrm{~mm}$ is the surface of a AUPA. When creating single high-pressure point at $(x, y, z)$, the phase of each transducer is delayed (controlled) following the phase delay factor $D$ [10]. $D$ is given by

$$
\begin{aligned}
D(x, y, z) & =\exp (-j k r), \\
r & =\sqrt{\left(x-x_{c}\right)^{2}+\left(y-y_{c}\right)^{2}+z^{2}},
\end{aligned}
$$

where $x_{c}$ and $y_{c}$ are the two-dimensional position of a transducer. $j$ is the imaginary unit and $k \mathrm{rad} / \mathrm{m}$ is the wavenumber.

The sound pressure presents a physical force named acoustic radiation force to a real object $P \mathrm{~Pa}$. $P$ is given by

$$
P=\frac{\alpha p^{2}}{\rho c^{2}},
$$

where $\alpha$ is a reflection coefficient of ultrasound, $\rho \mathrm{kg} / \mathrm{m}^{3}$ is a volume density of air, and $c \mathrm{~m} / \mathrm{s}$ is the sound velocity. 


\section{Mid-air Displays}
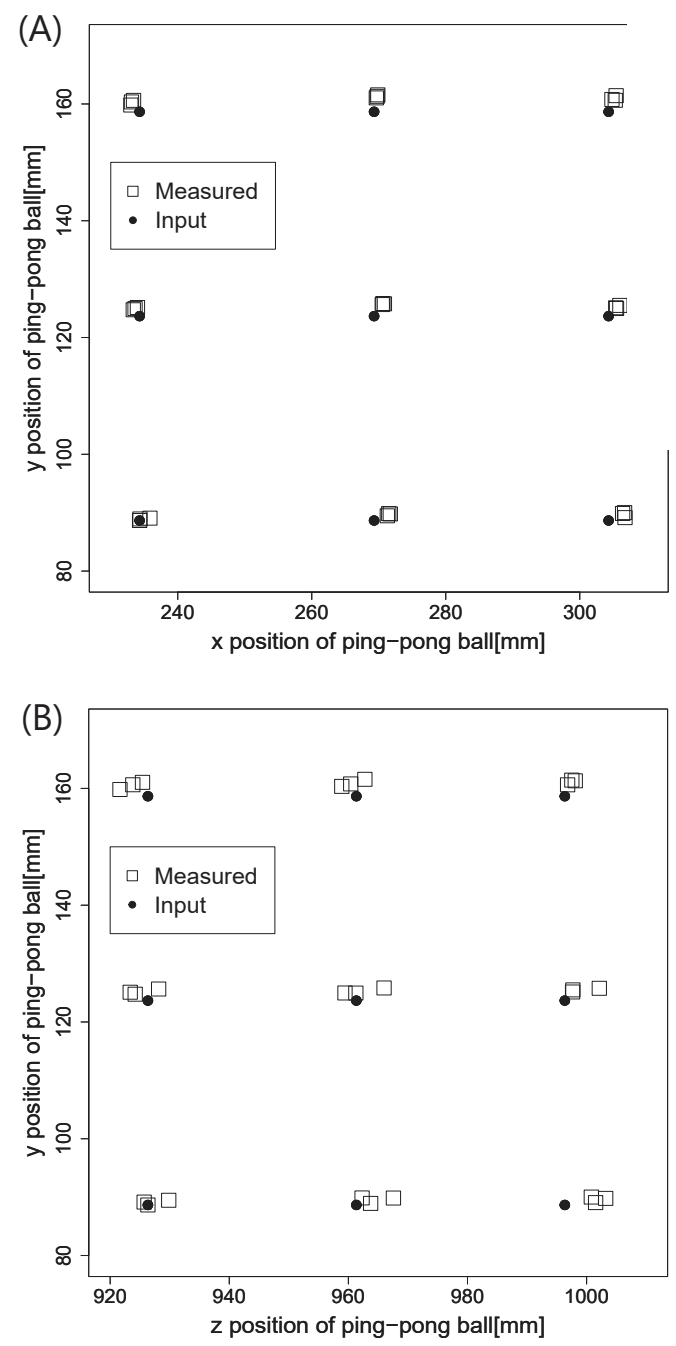

Figure 7. Tracking accuracy of ping-pong ball: (A) plotted on $x-y$ plane, (B) plotted on z-y plane.

Presenting force by ultrasound has a high spatial resolution 1 $\mathrm{cm}^{2} \times 3 \mathrm{~cm}$. Therefore, Hopping-Pong only pushes a PPB and does not injure players or audiences.

\section{Evaluation of presented force by AUPA}

We measured the force presented by nine AUPAs and evaluated whether the force can change the trajectory of a PPB or not.

Figure 9 shows the experimental setup. The radiation surfaces of AUPAs were facing downwards. In this experiment, AUPAs created a high-pressure point at the center of the nine AUPAs and a position of $300 \mathrm{~mm}, 450 \mathrm{~mm}$, and $600 \mathrm{~mm}$ from the surface. We measured the force of the high-pressure point with an electronic weighing instrument which could measure up to $620 \mathrm{~g}$ with the resolution of $0.001 \mathrm{~g}$.

Figure 10 shows a measurement result. From the result, we calculated the change of the trajectory of a PPB by AUPAs. Figure 11 shows a schematic illustration of the calculation. We simply assume that a PPB is always in the air when it passes
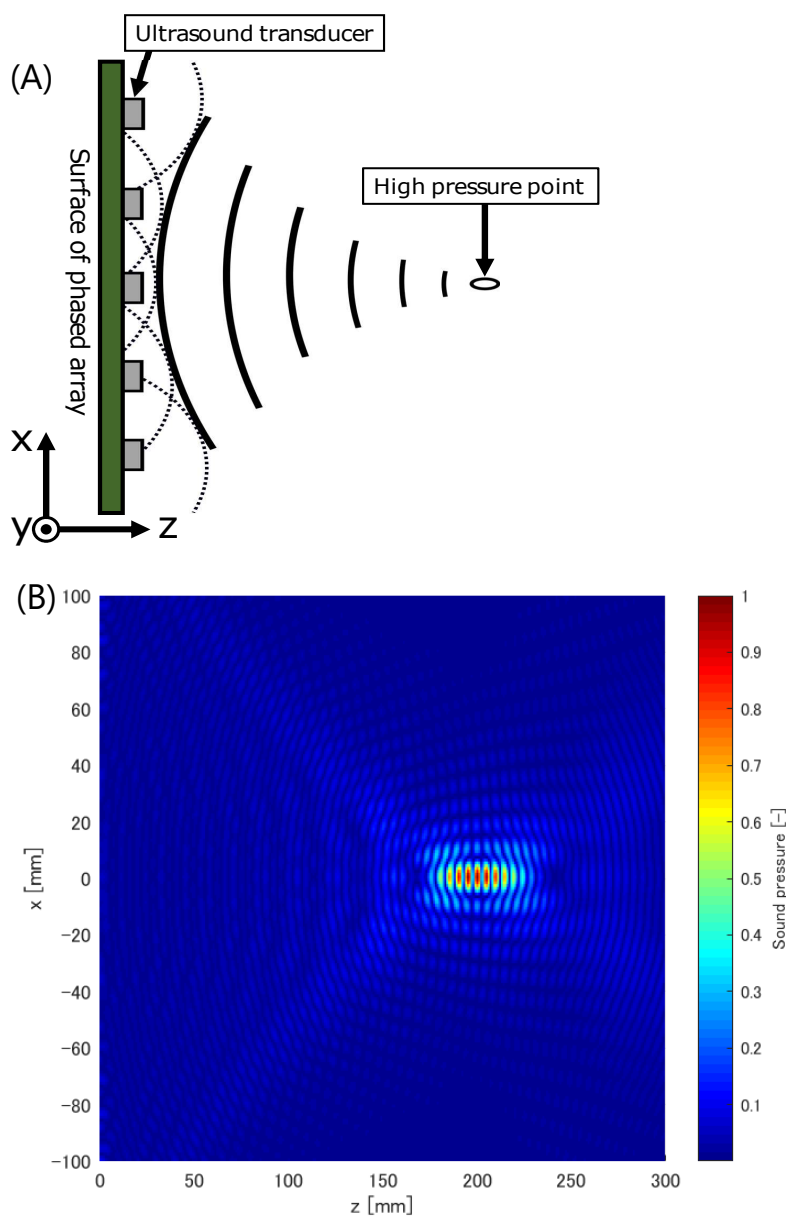

Figure 8. (A) Mechanism of creating small high-pressure point by airborne ultrasound phased array. (B) Distribution of effective sound pressure presented by airborne ultrasound phased array when creating a high-pressure point at $z=200 \mathbf{m m}$. The sound pressure is normalized.

the force presentable range. We define $t$ as elapsed time, $t_{0}$ as a time when a PPB is in the power presentation range, $t_{1}$ as a time when a PPB goes out of the range, and $t_{2}$ as a time when a PPB reaches the end of a table-tennis table. At $t_{0} \leq t \leq t_{1}$, the z-directional equation of motion of a PPB is as follows:

$$
F=m \frac{\mathrm{d}^{2} z}{\mathrm{~d} t^{2}}
$$

where $m \mathrm{~kg}$ is the mass of a PPB and $F \mathrm{~N}$ is a $\mathrm{z}$-directional external force presented by nine AUPAs. When assuming that the external force is applied constantly from $t_{0}$ to $t_{1}$, the $\mathrm{z}$-directional position of a PPB $z$ is given by solving eq. 4 under $z(0)=0$ and $\frac{\mathrm{d} z}{\mathrm{~d} t}(0)=0$ :

$$
z=\frac{F t^{2}}{2 m},
$$


Paper Session 3: Ultrasonic Techniques and Mid-air Displays

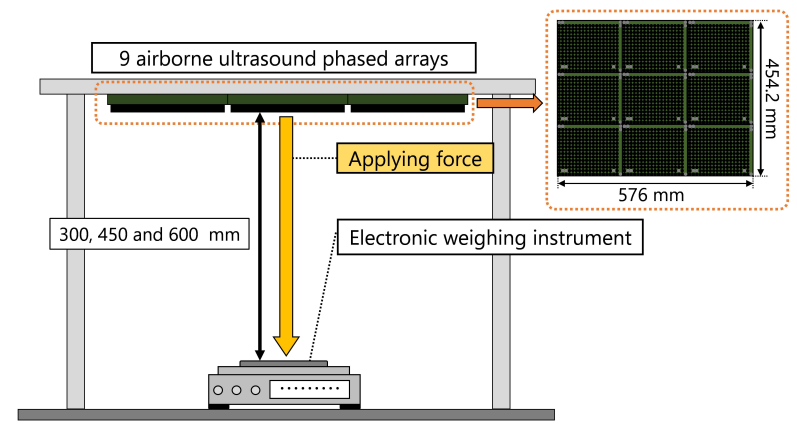

Figure 9. Experimental setup to measure total force of nine AUPAs.

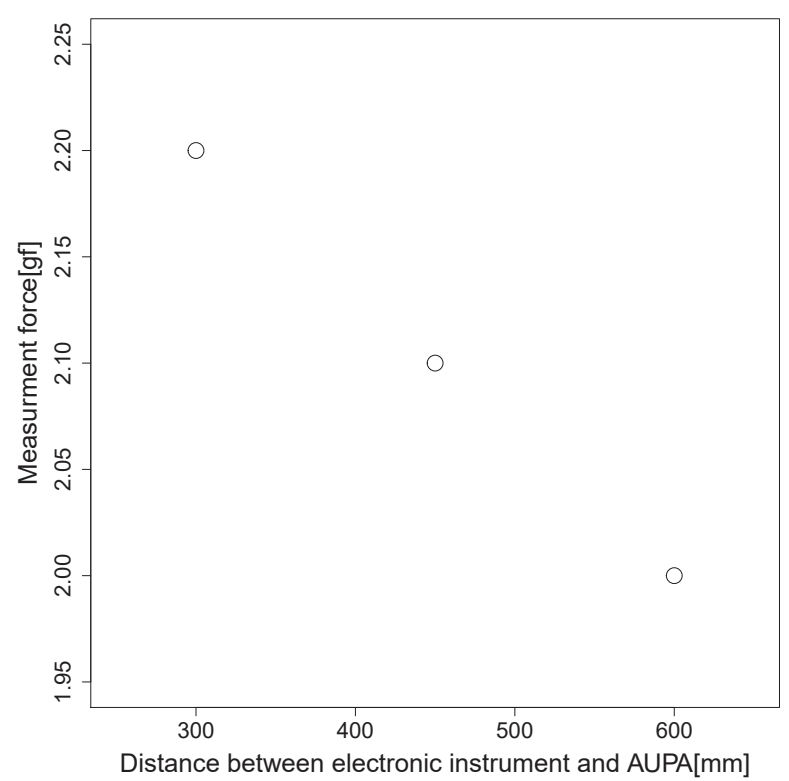

Figure 10. Total force of nine AUPAs measured by electronic weighing instrument.

which means that the PPB originally moves on the line $z=0$. From eq. $5, z$ at $t_{1} \leq t \leq t_{2}$ as follows:

$$
\begin{aligned}
v_{z} & =\frac{F\left(t_{1}-t_{0}\right)}{m}, \\
z & =v_{z}\left(t-t_{1}\right)+\frac{F\left(t_{1}-t_{0}\right)^{2}}{2 m} .
\end{aligned}
$$

To estimate a controllable distance by the system, we assumed each parameter as follows: velocity on the $\mathrm{x}$-axis of a PPB is $1 \mathrm{~m} / \mathrm{s}$. The length of a table-tennis table is $2.74 \mathrm{~m}$. The width of the power presentation range is $0.5 \mathrm{~m}, F=0.002 \times 9.8 \mathrm{~N}$ that is determined by the least value shown in Figure 10. The gravitational acceleration is $9.8 \mathrm{~m} / \mathrm{s}^{2}, m=2.4 \mathrm{~g}$, and $t_{0}=0$. Then, we get $t_{1}=0.5 \mathrm{~s}, t_{2}=1.37 \mathrm{~s}$ and $z\left(t_{2}\right)=4.57 \mathrm{~m}$ that is the final displacement from the original position $z=0$. This displacement is enough to augment the traditional ping-pong game.
ISS'19, November 10-13, 2019, Daejeon, Republic of Korea

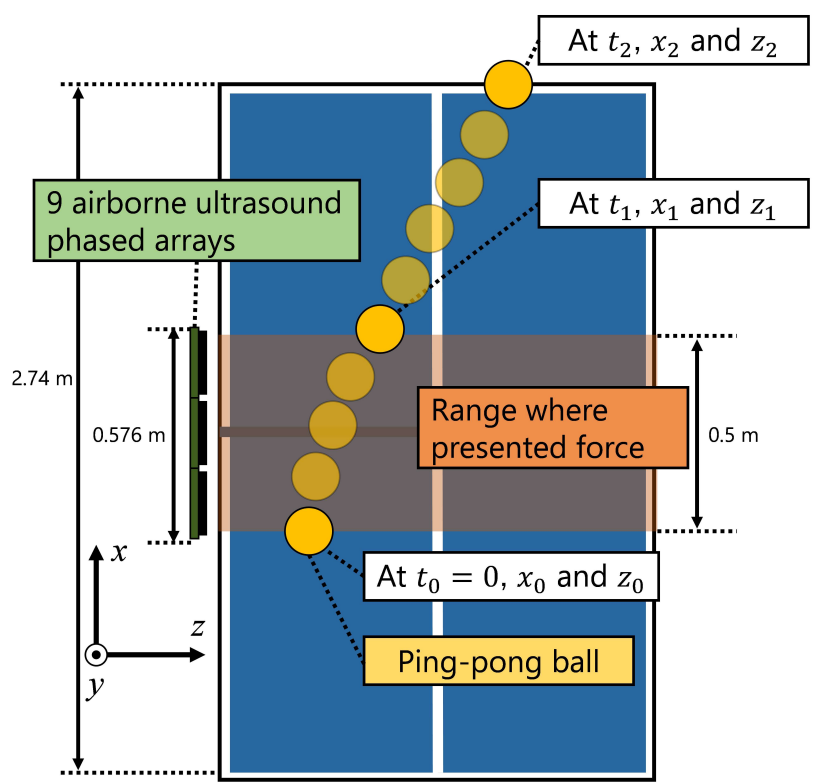

Figure 11. Schematic illustration for theoretical calculation of trajectory of ping-pong ball changed by Hopping-Pong.

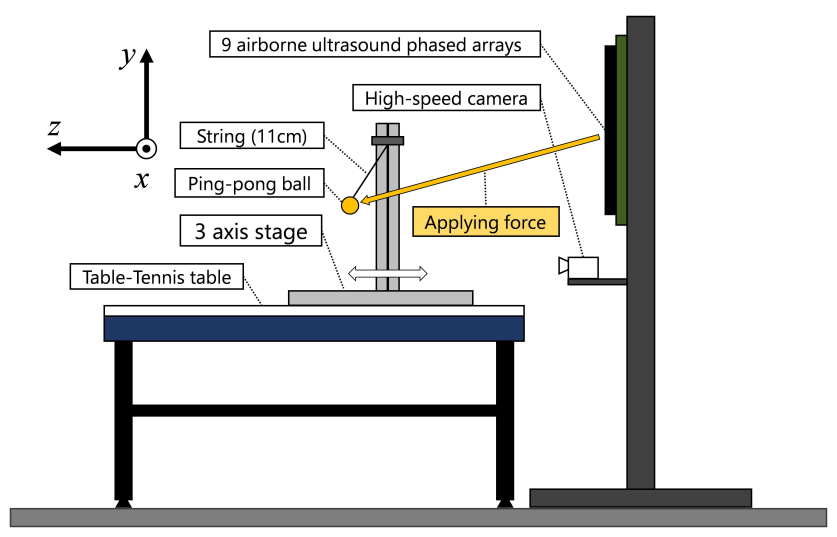

Figure 12. Experimental setup to measure distribution of force applied to ping-pong ball in Hopping-Pong.

\section{Evaluation of actual force along z-axis}

We measured and evaluated a force presented by nine AUPAs on the $\mathrm{z}$-axis under a realistic situation. Figure 12 shows the experimental setup. A PPB was suspended $11 \mathrm{~cm}$ below a three-axis stage with a thread. In this experiment, the PPB was tracked and stimulated by the Hopping-Pong system. To evaluate the actual given force onto a PPB, we measured the Z-directional moving distance of a PPB. By considering the equilibrium of forces, we calculated the distribution of the presented force along the $\mathrm{z}$-axis.

Figure 13 shows the measured $z$ position of the PPB and Figure 14 shows the calculated distribution of the presented force along the z-axis. In this calculation, the mass of the PPB was $2.4 \mathrm{~g}$ and the gravitational acceleration was $9.8 \mathrm{~m} / \mathrm{s}^{2}$. Furthermore, we assumed that the PPB only received $\mathrm{z}$-directional force from the AUPAs. The calculated force is lower than the 
Paper Session 3: Ultrasonic Techniques and Mid-air Displays

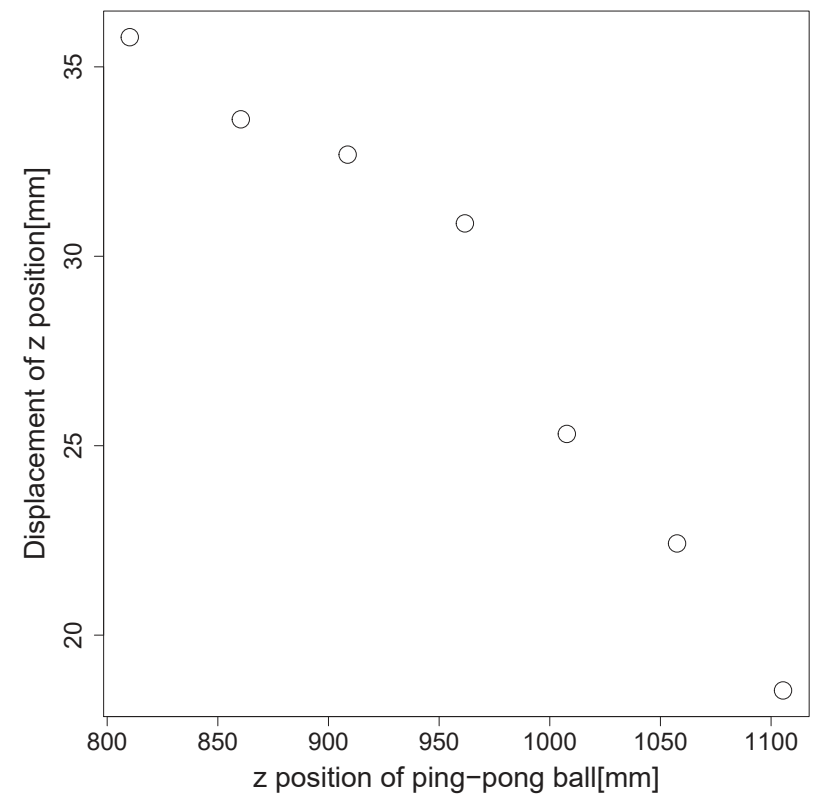

Figure 13. Displacement of ping-pong ball on z-axis.

force measured by an electronic weighing instrument shown in Figure 10. This is caused by the difference in the size of the area that receives force from the AUPAs. Because the area for the PPB is smaller than that of the electric weighing instrument, the total force could be different. When we assume that the given force is $0.5 \mathrm{gf}$, then the moving distance of $z\left(t_{2}\right)=1.14 \mathrm{~m}$, which is still sufficient for our purpose.

\section{Predicting Trajectory of Ping-Pong Ball}

In Hopping-Pong, there is a delay from capturing a PPB to presenting force. Therefore, Hopping-Pong predicts the trajectory of a PPB $16 \mathrm{~ms}$ ahead and presents force at the predicted position. In this section, we explain the cause of the delay and a prediction algorithm.

\section{Delay time in Hopping-Pong system}

There are three types of delays: 1) $d_{\text {cal }} \mathrm{s}$ is the time to calculate the position of a PPB, 2) $d_{\text {AUPA }} \mathrm{s}$ is a communication delay between a host PC and AUPA devices, and 3) $d_{\text {sound }} \mathrm{s}$ is the time for sound propagation from AUPAs to the target position. We confirmed that $d_{\text {cal }}$ is sufficiently shorter than the frame rate $\Delta t \mathrm{~s}$. Therefore, a position displacement of PPB on $\mathrm{x}$-axis caused by the delay that is as follows:

$$
\begin{aligned}
\Delta x & =v_{x}\left(d_{\mathrm{cal}}+d_{\mathrm{AUPA}}+d_{\text {sound }}\right), \\
d_{\text {sound }} & =\frac{z}{c},
\end{aligned}
$$

where $v_{x}$ is a velocity of a PPB on the $\mathrm{X}$-axis and $c \mathrm{~m} / \mathrm{s}$ is the sound velocity. In the system, $d_{\text {cal }}$ is $4 \mathrm{~ms}$ while $d_{\mathrm{AUPA}}$ cannot be measured directly. We empirically assumed that $d_{\mathrm{AUPA}}$ is $10 \mathrm{~ms}$ for estimating the difference of the position owing to the delay. We assumed other parameters as follows: $c$ is 340 $\mathrm{m} / \mathrm{s}, z$ is $0.5 \mathrm{~m}$ and $v_{x}$ is $2.74 \mathrm{~m} / \mathrm{s}$, where $2.74 \mathrm{~m} / \mathrm{s}$ is a velocity that passes a $2.74 \mathrm{~m}$ table-tennis table in 1 second. From eq.
ISS'19, November 10-13, 2019, Daejeon, Republic of Korea

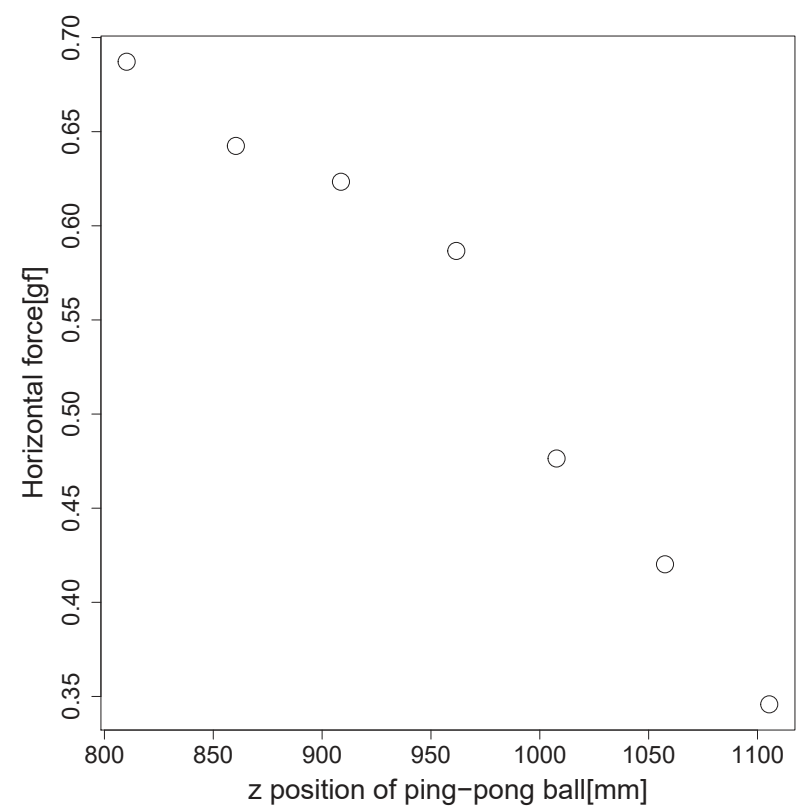

Figure 14. Actual force applied to ping-pong ball on z-axis. The force was calculated by the measured distribution shown in Figure 13.

8 , we calculated $\Delta x \sim 42 \mathrm{~mm}$. Since the radius of a PPB is $20 \mathrm{~mm}$ and the high-pressure point presented by AUPAs is $10 \mathrm{~mm}$ the high-pressure point cannot hit a PPB if we do not consider the delay.

Under the same conditions, we calculated the position displacement on the y-axis (vertical axis). We assumed that PPB is subjected by only gravity along the $y$-axis and moves upward at initial velocity $v_{y 0} \mathrm{~m} / \mathrm{s}$. We also assumed $\Delta y$ is constant within each frame $(\Delta t \mathrm{~s})$ and defined $\Delta y$ for each frame as $\Delta y_{i}(i=1,2,3 \ldots N) \mathrm{m} . \Delta y_{i}$ is given as follows:

$$
\begin{aligned}
\Delta y_{i} & =v_{y_{i}}\left(d_{\mathrm{cal}}+d_{\mathrm{AUPA}}+d_{\text {sound }}\right), \\
v_{y_{i}} & =-(i-1) g \Delta t+v_{y 0},
\end{aligned}
$$

where $v_{y_{i}}$ is a y-directional velocity of a PPB at $i$ frame and $g$ represents gravitational acceleration. At the vertex of parabolic motion, although $\Delta y_{i}$ is the minimum value, it is greater than $40 \mathrm{~mm}$ after $0.27 \mathrm{~s}$. Therefore, we also have to consider the delay effect in the y-direction to keep applying ultrasound force to a PPB.

\section{Prediction Algorithm}

Hopping-Pong predicts the trajectory of a PPB to compensate for the difference of $\Delta x$ and $\Delta y_{i}$. By assuming that a PPB moves linearly along the $\mathrm{X}$-axis, the estimated $\mathrm{x}$ position $\left(\hat{x}_{i}\right)$ is given as follows:

$$
\hat{x}_{i}=x_{i}+\frac{\left(x_{i}-x_{i-1}\right)}{\Delta t}\left(d_{\mathrm{cal}}+d_{\mathrm{AUPA}}+d_{\text {sound }}\right) .
$$

By assuming that the motion of a PPB on the y-axis is parabolic motion, a y position $\left(\hat{y}_{i}\right)$ is estimated by fitting a 
Paper Session 3: Ultrasonic Techniques and Mid-air Displays

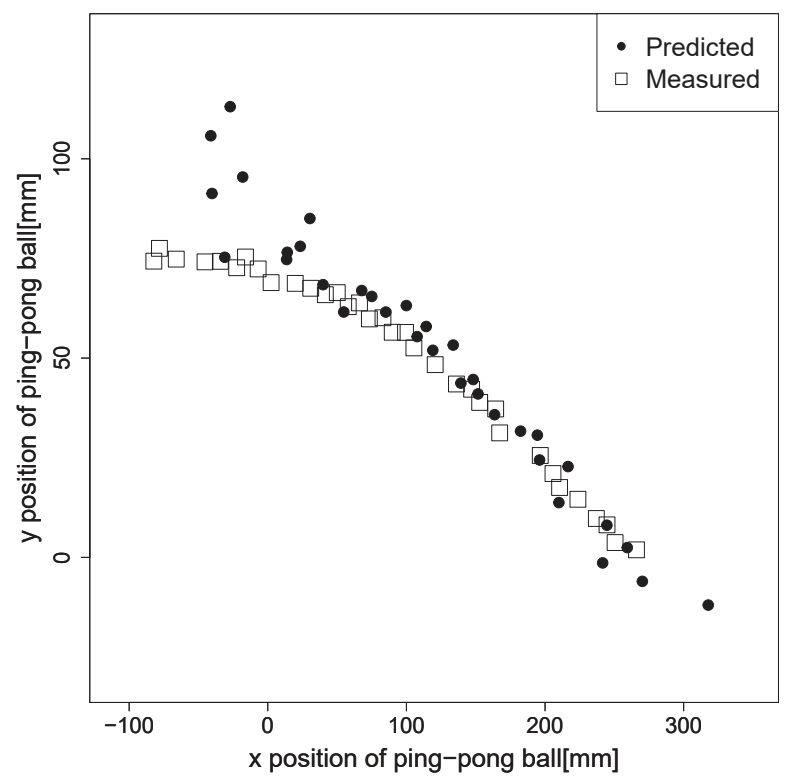

Figure 15. Predicted and measured trajectory of ping-pong ball.

quadratic curve to the measured data:

$$
\begin{aligned}
\hat{y_{i}=} & a_{i}\left(t_{i}+d_{\mathrm{cal}}+d_{\mathrm{AUPA}}+d_{\text {sound }}\right)^{2} \\
& +b_{i}\left(t_{i}+d_{\mathrm{cal}}+d_{\mathrm{AUPA}}+d_{\text {sound }}\right)+c_{i}, \\
t_{i}= & i \Delta t+T_{0},
\end{aligned}
$$

where $T_{0}$ is the time from when Hopping-Pong starts until a PPB is recognized. We analytically estimated the coefficients of $a_{i}, b_{i}$ and $c_{i}$ by using only the previous three positions of y to reduce the calculation time as follows:

$$
\begin{aligned}
a_{i}= & \frac{y_{i}\left(t_{\lceil i / 2\rceil}-t_{0}\right)+y_{\lceil i / 2\rceil}\left(t_{0}-t_{i}\right)+y_{0}\left(t_{i}-t_{\lceil i / 2\rceil}\right)}{\eta}, \\
b_{i}= & \frac{y_{i}\left(t_{0}^{2}-t_{\lceil i / 2\rceil}^{2}\right)+y_{\lceil i / 2\rceil}\left(t_{i}^{2}-t_{0}^{2}\right)+y_{0}\left(t_{\lceil i / 2\rceil}^{2}-t_{i}^{2}\right)}{\eta}(16) \\
c_{i}= & \frac{y_{i}\left(t_{\lceil i / 2\rceil}^{2} t_{0}-t_{0}^{2} t_{\lceil i / 2\rceil}\right)+y_{\lceil i / 2\rceil}\left(t_{0}^{2} t_{i}-t_{i}^{2} t_{0}\right)}{\eta} \\
& +\frac{y_{0}\left(t_{i}^{2} t_{0}-t_{0}^{2} t_{i}\right)}{\eta}, \\
\eta= & t_{\lceil i / 2\rceil}\left(t_{i}^{2}-t_{0}^{2}\right)+t_{0}\left(t_{\lceil i / 2\rceil}^{2}-t_{i}^{2}\right)+t_{i}\left(t_{0}^{2}-t_{\lceil i / 2\rceil}^{2}\right) .
\end{aligned}
$$

To improve the accuracy of the estimation, the three positions of $y$ were selected so that they are sufficiently far: $y_{i}, y_{\lceil i / 2\rceil}$ and $y_{0}$. Here, $y_{\lceil i / 2\rceil}=y_{(i+1) / 2}$ if the $i$ is an odd number.

Figure 15 shows the predicted trajectory of a PPB by eq. 12 and eq. 13 and the measured trajectory. The result shows that the algorithm can predict the trajectory of a PPB on the yaxis. The deviation of predicted values at the first nine frames is due to insufficient data. The trajectory of PPB must be convex upward parabolic, but we confirmed by eq. 13 that the predicted trajectory is convex downward at first nine frames.

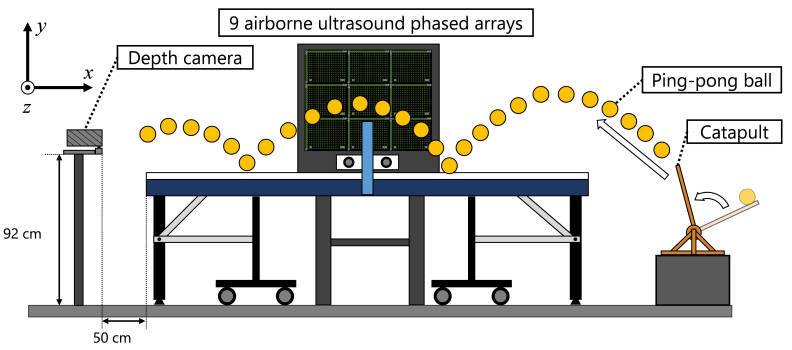

Figure 16. Experimental setup to measure displacement of ping-pong ball by Hopping-Pong.

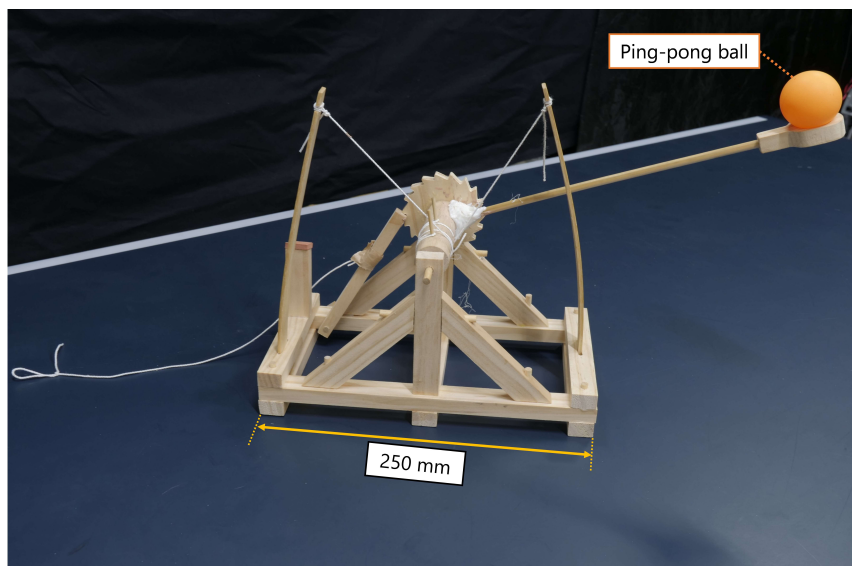

Figure 17. Catapult.

Since this deviation is only the first nine frames at Figure 15, there is no problem in practical use.

\section{PERFORMANCE EVALUATION}

We measured and evaluated the trajectory of a PPB changed by Hopping-Pong. Figure 16 shows the experimental setup. A PPB was thrown by a catapult made from wood as shown in Figure 17. The trajectory was measured by a depth camera (Kinect V2). The depth camera was installed approximately $0.5 \mathrm{~m}$ from the edge of the table-tennis table. The diameter of the used PPB is $40 \mathrm{~mm}$ and the weight is $2.4 \mathrm{~g}$. Then, we throw a PPB 10 times, with and without ultrasound force, respectively.

The measured trajectory is shown in Figure 18. In this experiment, the velocity of a PPB is $1.68 \mathrm{~m} / \mathrm{s}$ on average and the standard deviation (SD) is $0.29 \mathrm{~m} / \mathrm{s}$. The result with HoppingPong shows that the mean value of the PPB's z position at the end of the table-tennis table is $1078 \mathrm{~mm}$ and SD is $60 \mathrm{~mm}$. The result without Hopping-Pong shows that the mean value is $734 \mathrm{~mm}$ and the SD is $64 \mathrm{~mm}$. Therefore, Hopping-Pong can change the trajectory of a PPB by $344 \mathrm{~mm}$ on average. Assuming that the force is $0.5 \mathrm{gf}$, the same as the section of Distribution of presented force along $z$-axis, the theoretical displacement is $1.09 \mathrm{~m}$ at a PPB velocity of $1.68 \mathrm{~m} / \mathrm{s}$. Therefore, the measured displacement is $31.56 \%$ of the theoretical value. 


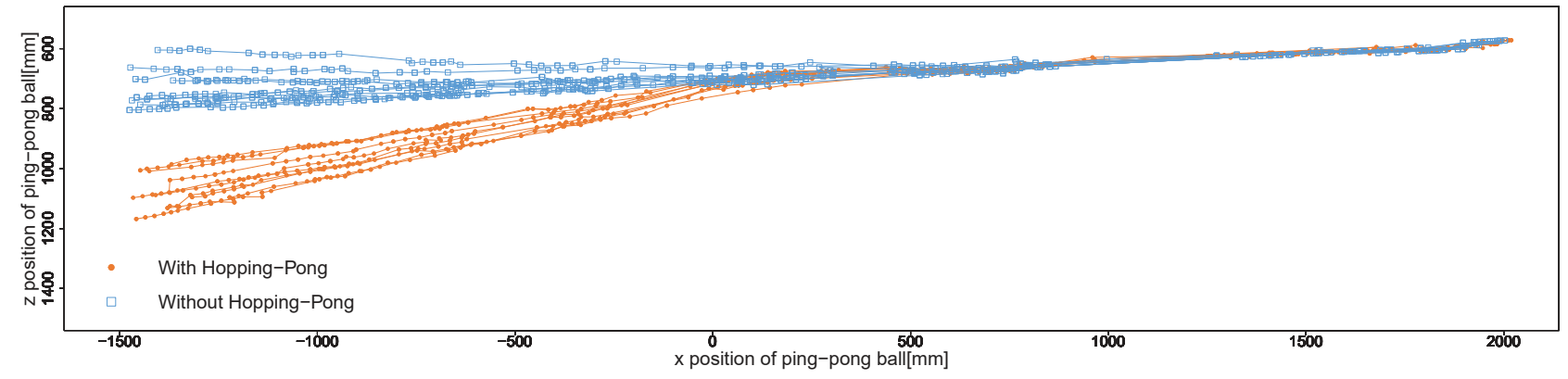

Figure 18. Displacement of trajectory of ping-pong ball with and without Hopping-Pong.

The displacement is more than twice the size of a general table-tennis racket. Therefore, the displacement of $344 \mathrm{~mm}$ is sufficient for a ping-pong game applications e.g. causing the player to fail to swing.

\section{DISCUSSION}

\section{Limitation and Future Work}

We discussed the capability and limitation of Hopping-Pong system to control a moving object that is our final goal. In this section, we focused on applying to a ping-pong game to specifically discuss and determine our next goal.

\section{Speed of moving object}

We conducted the performance evaluation at a ping-pong ball speed of $1.68 \mathrm{~m} / \mathrm{s}$. However, from the author's experience, when the speed became higher, Hopping-Pong could not change the trajectory of the PPB. $1.68 \mathrm{~m} / \mathrm{s}$ is not enough to play an actual ping-pong game. Our next step is the sufficient speed for a beginner ping-pong player.

We conjectured that there are two reasons for this limitation.

1. Hopping-Pong cannot correctly predict the trajectory of a high-speed PPB because $d_{\text {AUPA }}$ is not correctly determined.

2. The time for applying force is short owing to the narrower shooting range of high-speed cameras.

We consider that the first reason will be solved by a detailed analysis of the AUPA-PC communication system. The second reason will be solved by using a camera with a higher frame rate. We also conjecture that using more robust prediction methods e.g. Kalman filter can expand the camera shooting range without upgrading the capability of the camera. The number of measured positions of a PPB (frame rate) decreases when expanding the shooting range. If Hopping-Pong can predict the trajectory of a PPB by a robust prediction method from a few measurement positions, an ultrasound force can track the trajectory continuously with the low frame rate.

\section{Controllable direction}

At this stage, Hopping-Pong can only curve a moving PPB in only one direction. This is the first step for controlling moving objects via computational force. As the second step, we will control the displacement of the trajectory by closedloop control. Moreover, we will place the new AUPA unit on the other side to change the trajectory in the opposite direction.

\section{Safety of Hopping-Pong}

The safety of Hopping-Pong for a human is must be considered to apply for HCI. In this section, we described Hopping-Pong is sufficiently safe for human when applied for a ping-pong game. This discussion also implies that Hopping-Pong is safe in any situations.

We conjecture that Hopping-Pong does not injure human because the current AUPAs was initially developed as a tactile display for human [10]. Moreover, the ultrasound force degrades over the distance from the focus point and cannot affect objects outside a table tennis table.

The only potentially risky case is when all the following rare conditions are met: (1) the player's head enters the middle of a table tennis table, (2) the camera miss-recognizes the head as a PPB, and (3) the ultrasound focuses exactly on the entrance of the player's ear-hole. Players can even explicitly secure safely by merely wearing an earpiece.

\section{APPLICATIONS}

\section{Encouraging Motivation of Ping-Pong Beginners}

Our Hopping-Pong system can support beginners to shoot a curve-ball without requiring a high skill.

If given another set of AUPAs on the opposite side of the table, using closed-loop control and a motion sensor, this could realize advanced training among beginners. For example, the system may keep a PPB coming only in the backhand position. Moreover, Hopping-Pong can balance players in different skill levels so that both can enjoy playing together. For example, only the beginners can shoot the curve-ball; or Hopping-Pong always guides the ball near the beginners for an easy strike.

\section{For $\mathrm{HCl}$ and Practical Use}

The core technical advantage of the Hopping-Pong system is that it applies force in real-time to moving objects in a noncontact manner with high spatial resolution. We believe this unique capability is not limited to the ping-pong application and could open up various HCI use cases that can be of great importance in both science and industry. We include a few potential visionary applications from each discipline to illustrate our vision as follows. 


\section{Live specimen}

In neurophysiology, the Hopping-Pong principal may be of benefit for controlling small live specimens. In practical use, when changing genes of experimental insects such as drosophilas, observing their behavioral changes is necessary. Scientists usually fix the insects using a needle, but ultrasound may control this process without the risk of injuring insects. By tracking a small insect and using closed-loop control, HoppingPong can also control the trajectory of a fly in mid-air so that people can observe the natural behavior of the insect.

\section{Factory Lines}

In factory production lines, it is often necessary to remove defective samples. Especially in food production lines, defective or foreign matter should be removed by non-contact for the purposes of hygiene regulations. While typical systems use air-jet, the Hopping-Pong system can generate multiple high-pressure points at high spatial density without interfering with each other by devising the drive of phased array [5]. Thus, compared with air-jet that spreads, Hopping-Pong can remove a plurality of defective products simultaneously without affecting other good products.

\section{CONCLUSION}

In this paper, we explored the potential of computational force by applying the concept to a real moving object to change its trajectory. As a proof-of-concept system, we introduced Hopping-Pong, a system that changes the trajectory of a PPB flying in mid-air using a computationally controlled ultrasound force. Hopping-Pong system has technological contributions: 1) combining the high-speed measurement with the ultrasound force presentation while maintaining high frame rates (200 fps), 2) tracking and keeping applying force only a small moving object (PPB with a radius of $2 \mathrm{~cm}$ ) with a small force presentation area $\left(1 \mathrm{~cm}^{2} \times 3 \mathrm{~cm}\right)$.

Hopping-Pong could track a PPB with errors within $0.72 \mathrm{~mm}$ on the $\mathrm{x}$-axis, $0.76 \mathrm{~mm}$ on the $\mathrm{y}$-axis and $1.96 \mathrm{~mm}$ on the $\mathrm{z}$-axis. This was achieved using a high-speed camera and the change in the trajectory of a PPB by ultrasound, is approximately 344 $\mathrm{mm}$.

Based upon our results, we conclude that a computational force is capable of controlling not only a stationary object but also a moving object in the real world. This research contributes to expanding the real space that is controllable by computers and allows us to propose many applications in related fields such as augmented sports, HCI and factory automation technology.

\section{ACKNOWLEDGMENTS}

This work is supported in part by the JSPS Grant-in-Aid for Scientific Research 16H06303, JST CREST JPMJCR18A2, JST PRESTO 17939983, and JST PRESTO JPMJPR17J2.

\section{REFERENCES}

[1] Marco AB Andrade, Anne L Bernassau, and Julio C Adamowski. 2016. Acoustic levitation of a large solid sphere. Applied Physics Letters 109, 4 (2016), 044101.

[2] Marco AB Andrade, Fábio TA Okina, Anne L Bernassau, and Julio C Adamowski. 2017. Acoustic levitation of an object larger than the acoustic wavelength. The Journal of the Acoustical Society of America 141, 6 (2017), 4148-4154.

[3] Aaron Becker, Robert Sandheinrich, and Timothy Bretl. 2009. Automated manipulation of spherical objects in three dimensions using a gimbaled air jet. In 2009 IEEE/RSJ International Conference on Intelligent Robots and Systems. IEEE, 781-786.

[4] Peter J Berkelman, Ralph L Hollis, and David Baraff. 1999. Interaction with a real time dynamic environment simulation using a magnetic levitation haptic interface device. In Proceedings 1999 IEEE International Conference on Robotics and Automation (Cat. No. 99CH36288C), Vol. 4. IEEE, 3261-3266.

[5] Tom Carter, Sue Ann Seah, Benjamin Long, Bruce Drinkwater, and Sriram Subramanian. 2013. UltraHaptics: multi-point mid-air haptic feedback for touch surfaces. In Proceedings of the 26th annual ACM symposium on User interface software and technology. ACM, 505-514.

[6] Daniele Foresti, Majid Nabavi, Mirko Klingauf, Aldo Ferrari, and Dimos Poulikakos. 2013. Acoustophoretic contactless transport and handling of matter in air. Proceedings of the National Academy of Sciences 110, 31 (2013), 12549-12554.

[7] Euan Freeman, Julie Williamson, Sriram Subramanian, and Stephen Brewster. 2018. Point-and-shake: selecting from levitating object displays. In Proceedings of the 2018 CHI Conference on Human Factors in Computing Systems. ACM, 18.

[8] Takuro Furumoto, Masahiro Fujiwara, Yasutoshi Makino, and Hiroyuki Shinoda. 2019a. BaLuna: Floating Balloon Screen Manipulated Using Ultrasound. In Proceedings of the 26th IEEE Conference on Virtual Reality and 3D User Interfaces. IEEE, 221.

[9] Takuro Furumoto, Keisuke Hasegawa, Yasutoshi Makino, and Hiroyuki Shinoda. 2019b.

Three-Dimensional Manipulation of a Spherical Object Using Ultrasound Plane Waves. IEEE Robotics and Automation Letters 4, 1 (2019), 81-88.

[10] Takayuki Hoshi, Masafumi Takahashi, Takayuki Iwamoto, and Hiroyuki Shinoda. 2010. Noncontact tactile display based on radiation pressure of airborne ultrasound. IEEE Transactions on Haptics 3, 3 (2010), $155-165$.

[11] Seki Inoue, Yasutoshi Makino, and Hiroyuki Shinoda. 2016. Scalable architecture for airborne ultrasound tactile display. In International AsiaHaptics conference. Springer, 99-103.

[12] Seki Inoue, Shinichi Mogami, Tomohiro Ichiyama, Akihito Noda, Yasutoshi Makino, and Hiroyuki Shinoda. 2019. Acoustical boundary hologram for macroscopic rigid-body levitation. The Journal of the Acoustical Society of America 145, 1 (2019), 328-337. 
[13] Hiroshi Ishii, Craig Wisneski, Julian Orbanes, Ben Chun, and Joe Paradiso. 1999. PingPongPlus: design of an athletic-tangible interface for computer-supported cooperative play. In Proceedings of the SIGCHI conference on Human Factors in Computing Systems. ACM, 394-401.

[14] Yuta Itoh, Yuichi Hiroi, Jiu Otsuka, Maki Sugimoto, Jason Orlosky, Kiyoshi Kiyokawa, and Gudrun Klinker. 2016a. Laplacian vision: augmenting motion prediction via optical see-through head-mounted displays and projectors. In ACM SIGGRAPH 2016 Emerging Technologies. ACM, 13.

[15] Yuta Itoh, Jason Orlosky, Kiyoshi Kiyokawa, and Gudrun Klinker. 2016b. Laplacian vision: Augmenting motion prediction via optical see-through head-mounted displays. In Proceedings of the 7th Augmented Human International Conference 2016. ACM, 16.

[16] BV Jayawant. 1981. Electromagnetic suspension and levitation. Reports on Progress in Physics 44, 4 (1981), 411.

[17] Michinari Kono, Takayuki Hoshi, and Yasuaki Kakehi. 2014. Lapillus bug: Creature-like behaving particles based on interactive mid-air acoustic manipulation. In Proceedings of the 11th Conference on Advances in Computer Entertainment Technology. ACM, 34.

[18] Jinha Lee, Rehmi Post, and Hiroshi Ishii. 2011. ZeroN: mid-air tangible interaction enabled by computer controlled magnetic levitation. In Proceedings of the 24 th annual ACM symposium on User interface software and technology. ACM, 327-336.

[19] Mark Marshall, Thomas Carter, Jason Alexander, and Sriram Subramanian. 2012. Ultra-tangibles: creating movable tangible objects on interactive tables. In Proceedings of the SIGCHI Conference on Human Factors in Computing Systems. ACM, 2185-2188.

[20] Asier Marzo, Richard McGeehan, Jess McIntosh, Sue Ann Seah, and Sriram Subramanian. 2015. Ghost touch: Turning surfaces into interactive tangible canvases with focused ultrasound. In Proceedings of the 2015 International Conference on Interactive Tabletops \& Surfaces. ACM, 137-140.

[21] Florian'Floyd' Mueller and Martin R Gibbs. 2007a. Building a table tennis game for three players. In Proceedings of the international conference on Advances in computer entertainment technology. ACM, 179-182.

[22] Florian'Floyd' Mueller and Martin R Gibbs. 2007b. Evaluating a distributed physical leisure game for three players. In Proceedings of the 19th Australasian Conference on Computer-Human Interaction: Entertaining User Interfaces. ACM, 143-150.
[23] Kei Nitta, Keita Higuchi, and Jun Rekimoto. 2014. HoverBall: augmented sports with a flying ball. In Proceedings of the 5th Augmented Human International Conference. ACM, 13.

[24] Takuya Nojima, Ngoc Phuong, Takahiro Kai, Toshiki Sato, and Hideki Koike. 2015. Augmented dodgeball: an approach to designing augmented sports. In Proceedings of the 6th Augmented Human International Conference. ACM, 137-140.

[25] Yoichi Ochiai, Takayuki Hoshi, and Jun Rekimoto. 2014a. Pixie dust: graphics generated by levitated and animated objects in computational acoustic-potential field. ACM Transactions on Graphics (TOG) 33, 4 (2014), 85.

[26] Yoichi Ochiai, Takayuki Hoshi, and Jun Rekimoto. 2014b. Three-dimensional mid-air acoustic manipulation by ultrasonic phased arrays. PloS one 9, 5 (2014), e97590.

[27] Yuta Sugiura, Koki Toda, Takayuki Hoshi, Youichi Kamiyama, Takeo Igarashi, and Masahiko Inami. 2014. Graffiti fur: turning your carpet into a computer display. In Proceedings of the 27th annual ACM symposium on User interface software and technology. ACM, 149-156.

[28] Shun Suzuki, Ryoko Takahashi, Mitsuru Nakajima, Keisuke Hasegawa, Yasutoshi Makino, and Hiroyuki Shinoda. 2018. Midair Haptic Display to Human Upper Body. In 2018 57th Annual Conference of the Society of Instrument and Control Engineers of Japan (SICE). IEEE, 848-853.

[29] Yuki Uno, Hao Qiu, Toru Sai, Shunta Iguchi, Yota Mizutani, Takayuki Hoshi, Yoshihiro Kawahara, Yasuaki Kakehi, and Makoto Takamiya. 2018. Luciola: A millimeter-scale light-emitting particle moving in mid-air based on acoustic levitation and wireless powering. Proceedings of the ACM on Interactive, Mobile, Wearable and Ubiquitous Technologies 1, 4 (2018), 166.

[30] Chi Thanh Vi, Asier Marzo, Damien Ablart, Gianluca Memoli, Sriram Subramanian, Bruce Drinkwater, and Marianna Obrist. 2017. Tastyfloats: A contactless food delivery system. In Proceedings of the 2017 ACM International Conference on Interactive Surfaces and Spaces. ACM, 161-170.

[31] RR Whymark. 1975. Acoustic field positioning for containerless processing. Ultrasonics 13, 6 (1975), 251-261.

[32] Toshiya Yui and Tomoko Hashida. 2016. Floatio: Floating tangible user interface based on animacy perception. In Proceedings of the 29th Annual Symposium on User Interface Software and Technology. ACM, 43-45. 